\title{
Structural and Functional Investigation of FdhC from Acinetobacter nosocomialis: A Sugar N-Acyltransferase Belonging to the GNAT Superfamily
}

\author{
Ari J. Salinger, James B. Thoden, and Hazel M. Holden * \\ Department of Biochemistry, University of Wisconsin, Madison, Wisconsin 53706, United States
}

\begin{abstract}
Enzymes belonging to the GNAT superfamily are widely distributed in nature where they play key roles in the transfer of acyl groups from acyl-CoAs to primary amine acceptors. The amine acceptors run the gamut from histones to aminoglycoside antibiotics to small molecules such as serotonin. Whereas those family members that function on histones have been extensively studied, the GNAT enzymes that employ nucleotide-linked sugars as their substrates have not been well characterized. Indeed, though the structures of two of these "amino sugar" GNAT enzymes have been determined within the past 10 years, details concerning their active site architectures have been limited because of a lack of bound nucleotide-linked sugar substrates. Here we describe a combined structural and biochemical analysis of $\mathrm{FdhC}$ from Acinetobacter nosocomialis $\mathrm{O} 2$. On the basis of bioinformatics, it was postulated that FdhC catalyzes the transfer of a 3hydroxybutanoyl group from 3-hydroxylbutanoyl-CoA to dTDP-3-amino-3,6-dideoxy-D-galactose, to yield an unusual sugar that is ultimately incorporated into the surface polysaccharides of the bacterium. We present data confirming this activity. In addition, the structures of two ternary complexes of FdhC, in the presence of CoA and either 3-hydroxybutanoylamino-3,6-dideoxy-Dgalactose or 3-hydroxybutanoylamino-3,6-dideoxy-D-glucose, were solved by X-ray crystallographic analyses to high resolution. Kinetic parameters were determined, and activity assays demonstrated that FdhC can also utilize acetyl-CoA, 3-methylcrotonyl-CoA, or hexanoyl$\mathrm{CoA}$ as acyl donors, albeit at reduced rates. Site-directed mutagenesis experiments were conducted to probe the catalytic mechanism of FdhC. Taken together, the data presented herein provide significantly new molecular insight into those GNAT superfamily members that function on nucleotide-linked amino sugars.
\end{abstract}

\section{Graphical Abstract}

\footnotetext{
*Corresponding Author. Hazel_Holden@biochem.wisc.edu. Fax: 608-262-1319. Phone: 608-262-4988. ASSOCIATED CONTENT

Supporting Information

The Supporting Information is available free of charge on the ACS Publications website at DOI: 10.1021/acs.biochem.6b00602.

One table (PDF)
}

Accession Codes

X-ray coordinates have been deposited in the Research Collaboratory for Structural Bioinformatics, Rutgers University, New Brunswick, NJ (entries 5KTA, 5KTC, and 5KTD).

The authors declare no competing financial interest. 


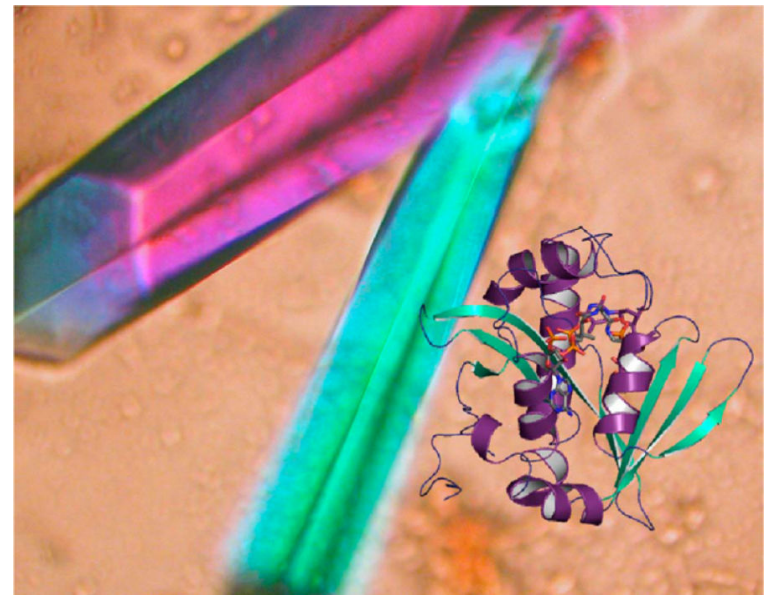

$N$-Acyltransferases are widespread in nature where they catalyze the transfer of acyl groups from acyl-CoAs to primary amine acceptors. Two structural superfamilies of $N$ acyltransferases have been identified thus far. One family contains the GCN5-related $N$ acetyltransferases, or GNAT enzymes, whereas the other is composed of the left-handed $\beta$ helix, or L $\beta \mathrm{H}$, enzymes. In recent years, our understanding of the $\mathrm{N}$-acyltransferases belonging to the $\mathrm{L} \beta \mathrm{H}$ superfamily, specifically those involved in the biosynthesis of unusual sugars, has dramatically improved as various structures have been reported. ${ }^{1-7}$ All of these enzymes contain a repeated isoleucine-rich, hexapeptide motif and a remarkable $\beta$-helix motif with exceedingly rare left-handed crossover connections.

Less is known, however, about those GNAT superfamily members that employ nucleotidelinked sugars as substrates. dTDP-fucosamine acetyltransferase (WecD) from Escherichia coli, which catalyzes the acetylation of dTDP-4-amino-4,6-dideoxy-D-galactose, was the first "amino sugar" GNAT superfamily member to have its structure determined. ${ }^{8}$ This initial report was followed by a hiatus for nine years until the structures of PseH from Campylobacter jejuni and Helicobacter pylori were solved. ${ }^{9,10}$ Whereas the WecD and PseH structures provided considerable molecular insight into the "nucleotide-linked sugar" GNAT superfamily, none of the available structures contained a substrate or substrate analogue.

Given our long-standing interest in enzymes involved in the biosynthesis of unique sugars found on the lipopolysaccharides of Gram-negative bacteria, and curious as to the manner in which a GNAT superfamily member can accommodate a nucleotide-linked sugar, we undertook an X-ray crystallographic analysis of the hypothetical protein $\mathrm{FdhC}$ from Acinetobacter nosocomialis $\mathrm{O} 2 .{ }^{11}$ Infections caused by such organisms as A. nosocomialis are becoming increasingly prevalent, and it is of particular concern that some isolates display resistance to all clinically available antibiotics. ${ }^{12}$ Although still a topic of debate, it is thought that Acinetobacter spp. produce lipooligosaccharides rather than lipopolysaccharides. Interestingly, the gene clusters required for the biosynthesis of the lipooligosaccharide sugars are extremely diverse in Acinetobacter spp. ${ }^{13}$ Some of the sugars in these organisms are quite unique, including 3-[(R)-3-hydroxybutanoylamino]-3,6dideoxy-D-galactose and 3-[(R)-3-hydroxybutanoylamino]-3,6-dideoxy-D-glucose, hereafter termed Fuc3N(R3Hb) and Qui3N(R3Hb), respectively. ${ }^{11}$ 
On the basis of its amino acid sequence, $A$. nosocomialis $\mathrm{FdhC}$ was predicted to catalyze the transfer of a 3-hydroxybutanoyl moiety from 3-hydroxylbutanoyl-CoA to dTDP-3amino-3,6-dideoxy-D-galactose (dTDP-Fuc3N) to yield dTDP-Fuc3N(R3Hb) as indicated in Scheme $1 .{ }^{11}$ Here we describe a combined structural and functional investigation of FdhC. For this analysis, three $\mathrm{X}$-ray structures were determined to high resolution, and kinetic parameters were measured. The kinetic data demonstrate that FdhC can function on both dTDP-Fuc3N and dTDP-3-amino-3,6-dideoxy-D-glucose (dTDP-Qui3N), albeit with significantly different catalytic efficiencies. In addition, two site-directed mutant variants, E131L and E131Q, were constructed to test the role of Glu 131 in catalysis. Importantly, two of the structures solved were those of the enzyme in complex with CoA and either dTDPFuc3N(R3Hb) or dTDP-Qui3N-(R3Hb). These models revealed for the first time the manner in which any GNAT superfamily member accommodates a nucleotide-linked sugar.

\section{MATERIALS AND METHODS}

\section{Cloning, Expression, and Purification}

The gene encoding FdhC from A. nosocomialis serogroup $\mathrm{O} 2$ was synthesized by DNA2.0 for optimized $E$. coli codon usage and was provided in a pJ201 plasmid with $5^{\prime}$-NdeI and $3^{\prime}$-XhoI restriction sites. The $f d h C$ gene was subsequently digested and ligated into a pET31b expression vector (Novagen) whereupon the STOP codon was removed to yield a protein with a C-terminal hexahistidine tag. For a tagless version of $\mathrm{FdhC}$, the $f d h C$ gene was ligated into pET28JT, a laboratory pET28b $(+)$ vector that had been previously modified to incorporate a TEV protease cleavage recognition site after the $\mathrm{N}$-terminal hexahistidine tag. ${ }^{14}$

Both vectors were utilized to transform Rosetta2(DE3) E. coli cells (Novagen). The cells harboring the pET31-fdhC plasmid were cultured in lysogeny broth supplemented with ampicillin $(100 \mathrm{mg} / \mathrm{L})$ and chloramphenicol $(25 \mathrm{mg} / \mathrm{L})$. The cells transformed with the pET28JT- $f d h C$ vector were cultured in lysogeny broth supplemented with kanamycin (35 $\mathrm{mg} / \mathrm{L})$ and chloramphenicol $(25 \mathrm{mg} / \mathrm{L})$. Both cell lines were grown while being shaken at $37^{\circ} \mathrm{C}$. When the optical density reached 0.8 at $600 \mathrm{~nm}$, the flasks were cooled in an ice/ water bath, and the cultures were subsequently induced with $1 \mathrm{mM}$ isopropyl $\beta$-D-1thiogalactopyranoside and transferred to a refrigerated shaker at $16^{\circ} \mathrm{C}$. The cells were allowed to express protein at $16{ }^{\circ} \mathrm{C}$ for $18 \mathrm{~h}$ after induction.

FdhC with the C-terminal tag was purified by standard procedures using Ni-nitrilotriacetic acid resin. Following purification, the protein was dialyzed against $10 \mathrm{mM}$ Tris- $\mathrm{HCl}$ and 200 $\mathrm{mM} \mathrm{NaCl}(\mathrm{pH} 8.0$ ) and concentrated to $18 \mathrm{mg} / \mathrm{mL}$ based on the calculated extinction coefficient of $0.62(\mathrm{mg} / \mathrm{mL})^{-1} \mathrm{~cm}^{-1}$. N-Terminally tagged FdhC was purified in a similar manner. For removal of the tag, a solution containing a 30:1 molar ratio (enzyme:TEV protease) was allowed to digest at $4{ }^{\circ} \mathrm{C}$ for $48 \mathrm{~h}$. Uncleaved protein and the TEV protease were removed by passage over Ni-nitrilotriacetic acid resin. Wild-type FdhC was dialyzed against $10 \mathrm{mM}$ Tris- $\mathrm{HCl}(\mathrm{pH} 8.0)$ and $200 \mathrm{mM} \mathrm{NaCl}$ and concentrated to $11 \mathrm{mg} / \mathrm{mL}$ on the basis of the calculated extinction coefficient of $0.62(\mathrm{mg} / \mathrm{mL})^{-1} \mathrm{~cm}^{-1}$. 
To express selenomethionine-labeled FdhC, the E. coli Rosetta2(DE3) cells containing the pET31-fdhC plasmid were grown in $\mathrm{M} 9$ minimal medium at $37^{\circ} \mathrm{C}$. Aliquots of $25 \mathrm{~mL}$ of this culture were then used to inoculate 12 baffled flasks $(2 \mathrm{~L}$ each) containing $1 \mathrm{~L}$ of M9 minimal medium supplemented with ampicillin $(100 \mathrm{mg} / \mathrm{L})$ and chloramphenicol $(50 \mathrm{mg} / \mathrm{L})$. The cultures were grown at $37{ }^{\circ} \mathrm{C}$ to an optical density of 0.9 at $600 \mathrm{~nm}$. Subsequently, the flasks were cooled on ice for $5 \mathrm{~min}$. L-Lysine, L-threonine, and L-phenylalanine $(50 \mathrm{mg} / \mathrm{L}$ each) and L-leucine, L-isoleucine, L-valine, and L-selenomethionine ( $25 \mathrm{mg} / \mathrm{L}$ each) were added to each flask. After being grown for an additional $20 \mathrm{~min}$, the cells were induced via the addition of $1 \mathrm{mM}$ isopropyl $\beta$-D-1-thiogalactopyranoside, and the protein was purified as described above. Mass spectrometry showed full incorporation of the selenium atoms.

\section{Site-Directed Mutagenesis}

All site-directed mutant variants of FdhC were generated via the QuikChange method of Stratagene. The protein variants were expressed and purified as described for the wild-type enzyme.

\section{Protein Crystallization and X-ray Structural Analysis}

Crystallization conditions were initially surveyed by the hanging drop method of vapor diffusion using a sparse matrix screen developed in the laboratory. The first experiments were conducted with either the apoprotein or a selenium-labeled form at room temperature. $\mathrm{X}$-ray diffraction quality crystals of both forms appeared as thin rods after 3 weeks and were grown by mixing in a $1: 1$ ratio the protein sample at $18 \mathrm{mg} / \mathrm{mL}$ with a precipitant solution that consisted of 2.4-2.8 M ammonium sulfate, 2\% dimethyl sulfoxide, and $100 \mathrm{mM}$ HEPPS (pH 8.5). The crystals grew to maximal dimensions of $1.0 \mathrm{~mm} \times 0.3 \mathrm{~mm} \times 0.3 \mathrm{~mm}$ and belonged to the space group $P 6_{3} 22$ with the following unit cell dimensions: $a=b=69.5 \AA$, and $c=137.9 \AA$. The asymmetric unit contained one subunit. Prior to X-ray data collection, the crystals of either the apoprotein or selenium-labeled enzyme were transferred to solutions that consisted of saturated ammonium sulfate, $2 \%$ dimethyl sulfoxide, $200 \mathrm{mM}$ $\mathrm{NaCl}, 100 \mathrm{mM}$ HEPPS (pH 8.5), and 10\% sucrose.

$\mathrm{X}$-ray diffraction quality crystals of $\mathrm{FdhC}$ with bound products were grown at $4{ }^{\circ} \mathrm{C}$ via batch experiments. Specifically, protein solutions containing $5 \mathrm{mM}$ 3-hydroxylbutanoyl-CoA and either $5 \mathrm{mM}$ dTDP-Fuc3N or dTDP-Qui3N substrate were mixed in a 1:1 ratio with precipitant solutions that consisted of 26-30\% monomethyl ether poly(ethylene glycol) 5000, $200 \mathrm{mM} \mathrm{LiCl}$, and $100 \mathrm{mM}$ Homo-PIPES (pH 5.0). The enzyme turned over the substrates during the crystallization experiments, and the products were trapped in the active site. Both complexes crystallized in the space group $P{ }_{1}{ }_{1}{ }_{1} 2$ with the following unit cell dimensions: $a=b=\sim 76 \AA$, and $c=73.5 \AA$. The asymmetric unit contained one monomer.

Crystals of the FdhC ternary complexes were flash-cooled for X-ray data collection via serial transfer into solutions that consisted of $25 \%$ monomethyl ether poly(ethylene glycol) $5000,300 \mathrm{mM} \mathrm{NaCl}, 100 \mathrm{mM} \mathrm{LiCl}, 100 \mathrm{mM}$ Homo-PIPES, and 16\% ethylene glycol (pH 5.0). The solutions also included each respective ligand at $5 \mathrm{mM}$.

X-ray data from the selenium-labeled protein crystals were collected at the Structural Biology Center beamline 19-BM at a wavelength of $0.9794 \AA$ (Advanced Photon Source). 
The X-ray data were processed and scaled with HKL3000. The structure was solved via multiple-wavelength anomalous dispersion phasing. The program CRANK from the CCP4 software suite was utilized to determine and refine the position of the single selenium atom. ${ }^{15}$ The preliminary electron density map allowed for a complete tracing of the polypeptide chain with the software package COOT. ${ }^{16}$ The model was partially refined to 2.1 A resolution with the software package REFMAC. ${ }^{17}$ This structure served as the search model for all subsequent $\mathrm{X}$-ray analyses.

$\mathrm{X}$-ray data sets from crystals of the apoprotein and the ternary complexes were collected in house at $100 \mathrm{~K}$ using a Bruker AXS Platinum $135 \mathrm{CCD}$ detector controlled with the Proteum software suite (Bruker AXS Inc.). The X-ray source was $\mathrm{Cu}$ Ka radiation from a Rigaku RU200 X-ray generator equipped with Montel optics and operated at $50 \mathrm{kV}$ and 90 $\mathrm{mA}$. These X-ray data were processed with SAINT version 7.06A (Bruker AXS Inc.) and internally scaled with SADABS version 2005/1 (Bruker AXS Inc.). The structures of the apoprotein and the ternary complexes were solved by molecular replacement using PHASER. ${ }^{18}$ The resulting models were subjected to alternating cycles of manual model building with COOT and refinement with REFMAC. Relevant X-ray data collection and model refinement statistics are provided in Tables 1 and 2, respectively.

\section{Size Exclusion Chromatography}

A Superdex 200 10/300 GL (GE Healthcare) column was equilibrated at room temperature with a buffer solution of $10 \mathrm{mM}$ Tris- $\mathrm{HCl}$ (pH 8.0) and $200 \mathrm{mM} \mathrm{NaCl}$ on an ÄKTA HPLC system. Purified C-terminally tagged protein (diluted to $10 \mathrm{mg} / \mathrm{mL}$ ) was applied to the column. The retention was monitored via ultraviolet absorption at $280 \mathrm{~nm}$. The results were compared to those of standards of known molecular mass.

\section{Activity of FdhC}

To confirm the $\mathrm{N}$-acyltransferase activity of FdhC, simple activity assays were first conducted. Specifically, $1 \mathrm{~mL}$ reaction mixtures were prepared using various CoA substrates (3-hydroxylbutanoyl-CoA, acetyl-CoA, 3-methylcrotonyl-CoA, or hexanoyl-CoA) and either dTDP-Fuc3N or dTDP-Qui3N. Each reaction condition included $1 \mathrm{mg} / \mathrm{mL}$ FdhC, 50 mM HEPPS ( $\mathrm{pH}$ 8.0), and $0.5 \mathrm{mM}$ CoA derivatives and dTDP-sugars. The reaction products were separated from the protein by filtration through a $10 \mathrm{kDa}$ cutoff ultrafiltration membrane, diluted with 2 volumes of water, and loaded onto an ÄKTA HPLC system equipped with a $1 \mathrm{~mL}$ Resource-Q column. Elution with a $20 \mathrm{~mL}$ gradient at $\mathrm{pH} 8.5$ from 0 to $0.6 \mathrm{M}$ ammonium bicarbonate showed the loss of the dTDP-sugar and CoA substrates (retention of 13.5 and $18 \mathrm{~mL}$, respectively) and the generation of a new peak with a retention volume of approximately $9 \mathrm{~mL}$. The identity of this peak as a modified dTDP-sugar was verified by electrospray ionization mass spectrometry (Mass Spectrometry/Proteomic Facility at the University of Wisconsin). The dTDP-Fuc3N and dTDP-Qui3N ligands required for the assays were prepared as previously described. ${ }^{19}$

\section{Determination of the Kinetic Constants of FdhC}

The $\mathrm{N}$-acyltransferase activity of FdhC was monitored spectrophotometrically by following the increase in absorbance at $412 \mathrm{~nm}$ due to the reaction of the free sulfhydryl group of the 
CoASH product with 5, $5^{\prime}$-dithiobis(2-nitrobenzoic acid) (DTNB). This reaction results in a disulfide interchange that leads to the formation of 5-thio-2-nitrobenzoic acid, which has a characteristic absorbance at $412 \mathrm{~nm}$ and an extinction coefficient of $14150 \mathrm{M}^{-1} \mathrm{~cm}^{-1}$. The use of this compound for quantification of free CoASH was first reported by Albers et al., ${ }^{20}$ and our assay method was similar to that described by Magalhaes et al. ${ }^{21}$ Reactions were continuously monitored with a Beckman DU 640B spectrophotometer. All reaction mixtures contained $50 \mathrm{mM}$ HEPES (pH 7.5) and $5 \mathrm{mM}$ DTNB in addition to enzyme and substrates.

For determination of the $K_{\mathrm{m}}$ of the enzyme for dTDP-Fuc3N, the concentration of 3hydroxylbutanoyl-CoA was held constant at $1 \mathrm{mM}$ while the dTDP-Fuc $3 \mathrm{~N}$ concentration varied from 0.02 to $5 \mathrm{mM}$. To measure the $K_{\mathrm{m}}$ of the enzyme for 3-hydroxylbutanoyl-CoA, the dTDP-Fuc3N concentration was held constant at $5 \mathrm{mM}$ while the 3-hydroxylbutanoylCoA concentration varied from 0.02 to $3 \mathrm{mM}$. All reactions were initiated by the addition of FdhC (or mutant variants) to a final concentration of $0.5 \mu \mathrm{g} / \mathrm{mL}$.

To measure the $K_{\mathrm{m}}$ of the enzyme for dTDP-Qui3N, the concentration of 3hydroxylbutanoyl-CoA was held constant at $1 \mathrm{mM}$ while the dTDP-Qui3N concentration varied from 0.02 to $10 \mathrm{mM}$. The reactions were initiated by the addition of FdhC to a final enzyme concentration of $0.5 \mu \mathrm{g} / \mathrm{mL}$.

Finally, for the determination of the $K_{\mathrm{m}}$ of the enzyme for acetyl-CoA, the dTDP-Fuc3N concentration was held constant at $5 \mathrm{mM}$ while the acetyl-CoA concentration varied from 0.002 to $1 \mathrm{mM}$. The final enzyme concentration was $0.5 \mathrm{mg} / \mathrm{mL}$.

All data were fitted by initial velocity Michaelis-Menten kinetics to the following equation:

$$
\nu=\left(V_{\max }[\mathrm{S}]\right) /\left(K_{\mathrm{m}}+[\mathrm{S}]\right)
$$

The $k_{\text {cat }}$ values were calculated according to the equation $k_{\text {cat }}=V_{\max } /[\mathrm{ET}]$. The reactions of FdhC with 3-methylcrotonyl-CoA and hexanoyl-CoA as substrates were too slow to be measured using the spectrophotometric assay. However, turnover was observed in the activity assays, and the identities of the products were confirmed via electrospray ionization mass spectrometry.

\section{RESULTS AND DISCUSSION}

\section{Kinetic Analysis of Wild-Type FdhC}

On the basis of bioinformatics, FdhC was proposed to function as an $\mathrm{N}$-acyltransferase, although this activity had never been biochemically verified. ${ }^{11}$ Thus, the first step in our enzymological analysis of FdhC was to conduct simple activity assays using 3hydroxylbutanoyl-CoA, acetyl-CoA, 3-methylcrotonyl-CoA, or hexanoyl-CoA as acyl donors and either dTDP-Qui3N or dTDP-Fuc3N as acyl acceptors. On the basis of electrospray ionization mass spectrometry of the FdhC products, it was shown that the enzyme could function on all of the CoA and dTDP-sugar substrates listed above (Figure S1). 
The next step was to determine the kinetic constants of the enzyme for these acyl donors and acceptors (Table 3). Consistent with the predicted function of FdhC, the catalytic efficiency of the enzyme for dTDP-Fuc3N versus dTDP-Qui3N was approximately 17 -fold higher. The catalytic efficiency of FdhC for acetyl-CoA, using dTDP-Fuc3N as the acceptor, was low at $8.5 \times 10 \mathrm{M}^{-1} \mathrm{~s}^{-1}$. Reactions with hexanoyl-CoA and 3-methylcrotonyl-CoA as the acyl donors were too slow to be kinetically analyzed under the assay conditions employed. Note that the protein utilized for the kinetic analyses did not contain a histidine tag. As described below, it was the C-terminal histidine-tagged version of the protein that crystallized. To ensure that the C-terminal tag did not affect the activity of the enzyme, the kinetic parameters for the tagged version were also determined as listed in Table 4 . The kinetic constants were similar for the two versions of FdhC.

\section{Overall Structure of FdhC}

The first structure to be determined in this investigation was that of the unliganded form of FdhC (with a C-terminal hexahistidine tag). The protein crystallized in the space group $P 6_{3} 22$ with one molecule in the asymmetric unit. The structure was determined to $1.9 \AA$ resolution, and the model was refined to an overall $R$ factor of $19.8 \%$. Although GNAT superfamily members typically function as dimers, there are clearly cases in which they assume monomeric quaternary structures. On the basis of both size exclusion gel filtration chromatography (Figure 1) as well as crystalline packing considerations, FdhC is a monomer in solution.

Shown in Figure 2 is a ribbon representation of the FdhC monomer. With overall dimensions of $\sim 47 \AA \times 49 \AA \times 43 \AA$, the FdhC architecture is quite compact. The polypeptide chain initiates with a $\beta$-hairpin motif, which is followed by two a-helices that are oriented nearly perpendicular to one another. The polypeptide chain then folds into a six-stranded mixed $\beta$ sheet flanked on one side by three $\alpha$-helices and on the other side by two $\alpha$-helices. As is typical of GNAT superfamily members, $\beta$-strands 3 and 4 of the mixed $\beta$-sheet splay apart. Electron density for the polypeptide chain backbone was continuous from Val 2 to Gly 179. Proline 89 adopts the cis conformation and lies in the loop connecting $\beta$-strands 2 and 3 (Figure 2). Two ordered sulfates and a dimethyl sulfoxide molecule were observed binding in the interior of the protein. The electron density for Cys 25 suggested that it had been oxidized to sulfenic acid.

\section{Structure of FdhC in Complex with CoA and either dTDP-Fuc3N(R3Hb) or dTDP- Qui3N(R3Hb)}

Crystals of the FdhC/CoA/dTDP-Fuc3N(R3Hb) complex belonged to the space group $P 4{ }_{1} 2{ }_{1} 2$, again with one molecule in the asymmetric unit. The crystals diffracted to a nominal resolution of $1.8 \AA$, and the overall $R$ factor for the final model was $19.7 \%$. Shown in Figure $3 \mathrm{a}$ is the observed electron density for the CoA and the dTDP-Fuc3N(R3Hb) ligands. The ordered sulfates and the dimethyl sulfoxide molecule observed in the apoenzyme structure are displaced by the dTDP-sugar. As can be seen, the pyranosyl moiety of the substrate adopts the ${ }^{4} \mathrm{C}_{1}$ conformation whereas the ribose assumes the $\mathrm{C} 2{ }^{\prime}$-endo pucker. A close-up view of the active site surrounding the dTDP-sugar ligand is depicted in Figure 3b. The thymine ring is anchored into the active site by the side chains of Gln 47 and Tyr 65 . In 
addition, it is sandwiched between the indole ring of $\operatorname{Trp} 50$ and the guanidinium group of $\operatorname{Arg} 28$, which forms a cation- $\pi$ stacking interaction. Two arginine residues, $\operatorname{Arg} 28$ and $\operatorname{Arg}$ 81 , interact with the $\beta$ - and a-phosphoryl groups of the dTDP-sugar, respectively. The side chain and backbone amide group of Ser 38 lie within $3.2 \AA$ of an a-phosphoryl and a $\beta$ phosphoryl oxygen, respectively. The only side chain that interacts with the pyranosyl moiety of the dTDP-sugar is Tyr 83 . Note that the $\mathrm{C}-4^{\prime}$ hydroxyl group lies within hydrogen bonding distance of only water molecules. The side chain of His 143 is situated within $3.2 \AA$ of the hydroxyl of the 3-hydroxybutanoyl group. There are no potential catalytic bases lying within $3.2 \AA$ of $\mathrm{N}-3^{\prime}$ of the dTDP-sugar. Glu 131 is the closest residue that could possibly function as a catalytic base via an ordered water molecule. This residue was targeted for further investigation as described below. The sulfur of the CoA sits at $3.6 \AA$ from the N-3' nitrogen.

Given the lack of protein interactions between the C-4' hydroxyl group of the dTDPFuc3N(R3Hb) product and the fact that the catalytic efficiency of FdhC with dTDP-Qui3N as a substrate is substantially reduced, the next structure determined in this investigation was that of FdhC complexed with CoA and dTDP-Qui3N(R3Hb). The model was refined at 1.9 $\AA$ resolution to an overall $R$ factor of $20.4 \%$. Shown in Figure 4a is the electron density observed for the bound ligands. Within experimental error, the two complex models are virtually identical such that their a-carbons superimpose with a root-mean-square deviation of $0.1 \AA$ A. A superposition of the active sites for these two models is displayed in Figure 4b. As can be seen, there are no major changes in the positions of either the ligands or the protein side chains. Indeed, the positions of the $\mathrm{C}-4^{\prime}$ atoms of the sugars differ by only 0.5 $\AA$ and those of the C-4' hydroxyl oxygens by $1.8 \AA$. The reason for the variations in catalytic efficiencies of FdhC for the two substrates is not obvious. Most likely, there are subtle differences that cannot be explained by time-averaged crystal structures.

Shown in Figure 5 is a superposition of the ribbon drawings for the apoenzyme and the FdhC/CoA/dTDP-Fuc3N(R3Hb) complex. Excluding the loop defined by Asn 30 to Asp 43, the a-carbons for these two models superimpose with a root-meansquare deviation of $0.5 \AA$. The Asn 30-Asp 43 loop, which connects the first two a-helices of the polypeptide chain, begins to diverge between the two models at Asn 29. Indeed, there are significant changes in the dihedral angles for Asn 29 (for the apoenzyme, $\phi=-66^{\circ}$ and $\psi=-56^{\circ}$; for the ternary complex, $\phi=-100^{\circ}$ and $\psi=7.8^{\circ}$ ). As a consequence, some of the a-carbons moved by as much as $\sim 13 \AA$ when ligands bind to the FdhC active site. The driving force for this loop closure is apparently Ser 38, which forms hydrogen bonding interactions with both the aand $\beta$-phosphoryl groups of the dTDP-sugar ligand through its side chain and its backbone amide group, respectively (Figure $3 b$ ). Although caution must be used in the interpretation of $B$ values, it is noteworthy that the average $B$ factors for the loops in the apoenzyme and the ternary complex models are 48 and $24 \AA^{2}$, respectively. Whereas it cannot be ruled out that this loop movement is simply an artifact of crystal packing, it does suggest protein flexibility in this region. This type of movement is also not without precedence in the $N$ acetyltransferases. For example, in the apo and liganded structures of serotonin $N$ acetyltransferase, there is a large rearrangement of the loop connecting the first two ahelices of the polypeptide chain, as well. ${ }^{22}$ 
FdhC has an overall molecular architecture similar to that observed for PseH from $C$. jejuni and $H$. pylori and WecD from E. coli. The following comparison will focus on PseH from $H$. pylori given that it is similar in size to FdhC and that the X-ray model for the protein had no breaks in the polypeptide chain. PseH catalyzes the $N$-acetylation of UDP-4-amino-4,6dideoxy- $N$-acetyl-L-altrosamine, and thus, it functions on a C- $4^{\prime}$ rather than $\mathrm{C}-3^{\prime}$ amino group. Overall, the amino acid sequence identity and similarity between $\mathrm{FdhC}$ and $\mathrm{PseH}$ are quite low at 21 and 37\%, respectively. A superposition of the ribbon representations for these two enzymes is presented in Figure 6. As can be seen, the overall folds of the two enzymes are strikingly similar. Indeed, the a-carbons for these two proteins superimpose with a root-mean-square deviation of $1.6 \AA$ for 150 target pairs. The structure of PseH was solved in the presence of acetyl-CoA. The $4^{\prime}$-phosphopantetheine units align well between the two proteins. The adenosine groups, however, are oriented at nearly $180^{\circ}$ from one another. Although the structure of PseH was solved in the absence of a UDP-sugar ligand, on the basis of the superposition shown in Figure 6, it can be postulated that, similar to FdhC, the side chains of Arg 30 and Phe 52 abut either side of the uracil ring. The equivalent residues in FdhC are Arg 28 and Trp 50. Likewise, in FdhC, the side chains of Gln 47 and Tyr 65 form hydrogen bonding interactions with the thymine ring of the substrate. In PseH, the structurally equivalent residues are His 49 and Tyr 64, which might also serve a role in nucleobase positioning. As noted above, Ser 38 in FdhC serves to anchor the phosphoryl groups of the dTDP-sugar substrate into the active site. It is possible that Tyr 40 functions in a similar manner in PseH. A more detailed comparison of the active sites of these two enzymes will require an $\mathrm{X}$-ray analysis of $\mathrm{PseH}$ as a ternary complex.

\section{Catalytic Mechanism of FdhC}

There has been considerable debate in the literature regarding the catalytic mechanisms of GNAT superfamily members and the need for a catalytic base to remove a proton from the amino group that is ultimately acylated. ${ }^{23-25}$ Inspection of the FdhC active site revealed no side chains located within $\sim 3.2 \AA$ that could function as a catalytic base. There was an ordered water molecule, however, located near the C- $3^{\prime}$ nitrogens of the dTDP-sugar ligands in both ternary complex models. As can be seen in Figure 3b, this solvent is positioned between the $\mathrm{C}-3^{\prime}$ nitrogen of the dTDP-sugar substrate and the carboxylate of Glu 131. To test whether a water-mediated proton relay was playing a role in FdhC catalysis, two site-directed variants were constructed and kinetically studied. Both E131Q and E131L were able to turn over the substrates, and in fact, their catalytic efficiencies were not significantly altered (Table 4). Clearly, from the site-directed mutagenesis experiments, Glu 131 does not function as a catalytic base.

Although it can be argued that at physiological $\mathrm{pH}$ the $\mathrm{C}-3^{\prime}$ amino group of the FdhC substrate would be positively charged, the hydrophobic nature of the active site surrounding the pyranosyl group suggests that the neutral amino sugar is bound. A catalytic mechanism can thus be envisioned whereby the neutral amine functions as the nucleophile that attacks the carbonyl carbon of acyl-CoA. This attack leads to a cationic intermediate that spontaneously deprotonates. In addition, the thiolate of CoA is an excellent leaving group so there may be no need for a catalytic acid. The reaction mechanism of FdhC, as well as 
perhaps many other GNAT superfamily members, most likely proceeds via catalysis by approximation.

In summary, the research described here demonstrates that $\mathrm{FdhC}$ is, indeed, an $N$ acyltransferase as originally suggested on the basis of bioinformatics. Importantly, for the first time, the structure of a GNAT superfamily member that functions on a nucleotide-linked sugar has been obtained as a ternary complex. As a consequence, key interactions between the dTDP-sugar and the protein have been identified. This study thus provides new molecular insight into the GNAT superfamily in general.

\section{Supplementary Material}

Refer to Web version on PubMed Central for supplementary material.

\section{Acknowledgments}

A portion of the research described in this paper was performed at Argonne National Laboratory, Structural Biology Center, at the Advanced Source (U.S. Department of Energy, Office of Biological and Environmental Research, under Contract DE-AC02-06CH11357). We gratefully acknowledge Dr. Norma E. C. Duke and Dr. Stephan L. Ginell for assistance during the X-ray data collection at Argonne.

Funding

This research was supported in part by National Institutes of Health Grant GM115921 (to H.M.H.).

\section{ABBREVIATIONS}

$\begin{array}{ll}\text { CoA } & \text { coenzyme A } \\ \text { DTNB } & 5,5^{\prime} \text {-dithiobis(nitrobenzoic acid) } \\ \text { dTDP-Fuc3N } & \text { dTDP-3-amino-3,6-dideoxy-D-galactose } \\ \text { dTDP-Qui3N } & \text { dTDP-3-amino-3,6-dideoxy-D-glucose } \\ \text { Fuc3N(R3Hb) } & 3 \text {-[(R)-3-hydroxybutanoylamino]-3,6-dideoxy-D-galactose } \\ \text { Qui3N(R3Hb) } & 3-[(R)-3 \text {-hydroxybutanoylamino }]-3,6 \text {-dideoxy-D-glucose } \\ \text { HEPES } & N \text {-(2-hydroxyethyl)piperazine- } N^{\prime}-2 \text {-ethane-sulfonic acid } \\ \text { HEPPS } & N \text {-(2-hydroxyethyl)piperazine- } N N^{\prime}-3 \text {-propanesulfonic acid } \\ \text { Homo-PIPES } & \text { homopiperazine-1,4-bis-(2-ethanesulfonic acid) } \\ \text { HPLC } & \text { high-performance liquid chromatography } \\ \text { TEV } & \text { tobacco etch virus } \\ \text { Tris } & \text { tris-(hydroxymethyl)aminomethane }\end{array}$




\section{REFERENCES}

1. Rangarajan ES, Ruane KM, Sulea T, Watson DC, Proteau A, Leclerc S, Cygler M, Matte A, Young NM. Structure and active site residues of PgID, an $N$-acetyltransferase from the bacillosamine synthetic pathway required for $\mathrm{N}$-glycan synthesis in Campylobacter jejuni. Biochemistry. 2008; 47:1827-1836. [PubMed: 18198901]

2. Olivier NB, Imperiali B. Crystal structure and catalytic mechanism of PglD from Campylobacter jejuni. J. Biol. Chem. 2008; 283:27937-27946. [PubMed: 18667421]

3. Thoden JB, Cook PD, Schaffer C, Messner P, Holden HM. Structural and functional studies of QdtC: an $N$-acetyltransferase required for the biosynthesis of dTDP-3-acetamido-3,6-dideoxyalpha-d-glucose. Biochemistry. 2009; 48:2699-2709. [PubMed: 19191736]

4. Chantigian DP, Thoden JB, Holden HM. Structural and biochemical characterization of a bifunctional ketoisomerase/ $\mathrm{N}$-acetyltransferase from Shewanella denitrif icans. Biochemistry. 2013; 52:8374-8385. [PubMed: 24128043]

5. Thoden JB, Reinhardt LA, Cook PD, Menden P, Cleland WW, Holden HM. Catalytic mechanism of perosamine $\mathrm{N}$-acetyltransferase revealed by high-resolution X-ray crystallographic studies and kinetic analyses. Biochemistry. 2012; 51:3433-3444. [PubMed: 22443398]

6. Kubiak RL, Holden HM. Structural studies of AntD: an $N$-Acyltransferase involved in the biosynthesis ofd-anthrose. Biochemistry. 2012; 51:867-878. [PubMed: 22220494]

7. Thoden JB, Holden HM. Molecular structure of WlbB, a bacterial $N$-acetyltransferase involved in the biosynthesis of 2,3-diacetamido-2,3-dideoxy-d-mannuronic acid. Biochemistry. 2010; 49:46444653. [PubMed: 20433200]

8. Hung MN, Rangarajan E, Munger C, Nadeau G, Sulea T, Matte A. Crystal structure of TDPfucosamine acetyltransferase (WecD) from Escherichia coli an enzyme required for enterobacterial common antigen synthesis. J. Bacteriol. 2006; 188:5606-5617. [PubMed: 16855251]

9. Song WS, Nam MS, Namgung B, Yoon SI. Structural analysis of PseH, the Campylobacter jejuni $N$ acetyltransferase involved in bacterial $O$-linked glycosylation. Biochem. Biophys. Res. Commun. 2015; 458:843-848. [PubMed: 25698400]

10. Ud-Din AI, Liu YC, Roujeinikova A. Crystal structure of Helicobacter pylori pseudaminic acid biosynthesis $\mathrm{N}$-acetyltransferase PseH: implications for substrate specificity and catalysis. PLoS One. 2015; 10:e0115634. [PubMed: 25781966]

11. Hu D, Liu B, Dijkshoorn L, Wang L, Reeves PR. Diversity in the major polysaccharide antigen of Acinetobacter baumannii assessed by DNA sequencing, and development of a molecular serotyping scheme. PLoS One. 2013; 8:e70329. [PubMed: 23922982]

12. Weber BS, Harding CM, Feldman MF. Pathogenic Acinetobacter: from the Cell Surface to Infinity and Beyond. J. Bacteriol. 2016; 198:880-887.

13. Kenyon JJ, Nigro SJ, Hall RM. Variation in the OC locus of Acinetobacter baumannii genomes predicts extensive structural diversity in the lipooligosaccharide. PLoS One. 2014; 9:e107833. [PubMed: 25247305]

14. Thoden JB, Holden HM. The molecular architecture of human $\mathrm{N}$-acetylgalactosamine kinase. J. Biol. Chem. 2005; 280:32784-32791. [PubMed: 16006554]

15. Ness SR, de Graaff RA, Abrahams JP, Pannu NS. CRANK: new methods for automated macromolecular crystal structure solution. Structure. 2004; 12:1753-1761. [PubMed: 15458625]

16. Emsley P, Cowtan K. Coot: model-building tools for molecular graphics. Acta Crystallogr., Sect. D: Biol. Crystallogr. 2004; 60:2126-2132. [PubMed: 15572765]

17. Murshudov GN, Vagin AA, Dodson EJ. Refinement of macromolecular structures by the maximum-likelihood method. Acta Crystallogr., Sect. D: Biol. Crystallogr. 1997; 53:240-255. [PubMed: 15299926]

18. McCoy AJ, Grosse-Kunstleve RW, Adams PD, Winn MD, Storoni LC, Read RJ. Phaser crystallographic software. J. Appl. Crystallogr. 2007; 40:658-674. [PubMed: 19461840]

19. Woodford CR, Thoden JB, Holden HM. New role for the ankyrin repeat revealed by a study of the $N$-formyltransferase from Providencia alcalifaciens. Biochemistry. 2015; 54:631-638. [PubMed: 25574689] 
20. Alpers DH, Appel SH, Tomkins GM. A spectrophotometric assay for thiogalactoside transacetylase. J. Biol. Chem. 1965; 240:10-13. [PubMed: 14253400]

21. Magalhaes ML, Blanchard JS. The kinetic mechanism of AAC3-IV aminoglycoside acetyltransferase from Escherichia coli. Biochemistry. 2005; 44:16275-16283. [PubMed: 16331988]

22. Hickman AB, Namboodiri MA, Klein DC, Dyda F. The structural basis of ordered substrate binding by serotonin $N$-acetyltransferase: enzyme complex at 1.8 A resolution with a bisubstrate analog. Cell. 1999; 97:361-369. [PubMed: 10319816]

23. Dyda F, Klein DC, Hickman AB. GCN5-related $N$-acetyltransferases: a structural overview. Annu. Rev. Biophys. Biomol. Struct. 2000; 29:81-103. [PubMed: 10940244]

24. Vetting MW, S. de Carvalho LP, Yu M, Hegde SS, Magnet S, Roderick SL, Blanchard JS. Structure and functions of the GNAT superfamily of acetyltransferases. Arch. Biochem. Biophys. 2005; 433:212-226. [PubMed: 15581578]

25. Favrot L, Blanchard JS, Vergnolle O. Bacterial GCN5-Related $N$-acetyltransferases: from resistance to regulation. Biochemistry. 2016; 55:989-1002. [PubMed: 26818562]

26. Laskowski RA, Moss DS, Thornton JM. Main-chain bond lengths and bond angles in protein structures. J. Mol. Biol. 1993; 231:1049-1067. [PubMed: 8515464]

27. DeLano, WL. The PyMOL Molecular Graphics System. San Carlos, CA: DeLano Scientific; 2002. 


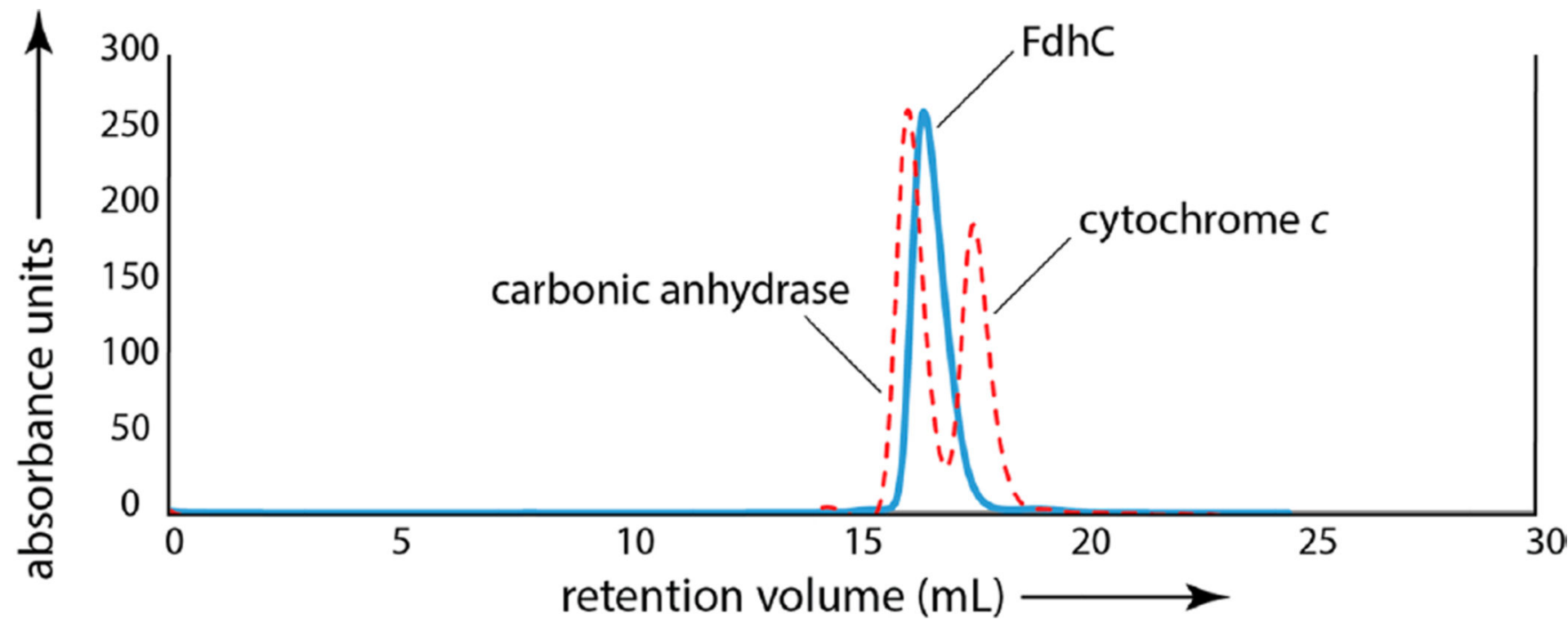

Figure 1.

Gel filtration chromatography of FdhC. Shown in the blue trace is the retention volume of the FdhC sample vs absorbance. The red dashed lines show the retention times for horse heart cytochrome $c$ (molecular weight of $~ 12400$ ) and bovine erythrocyte carbonic anhydrase (molecular weight of $\sim 29000$ ). 

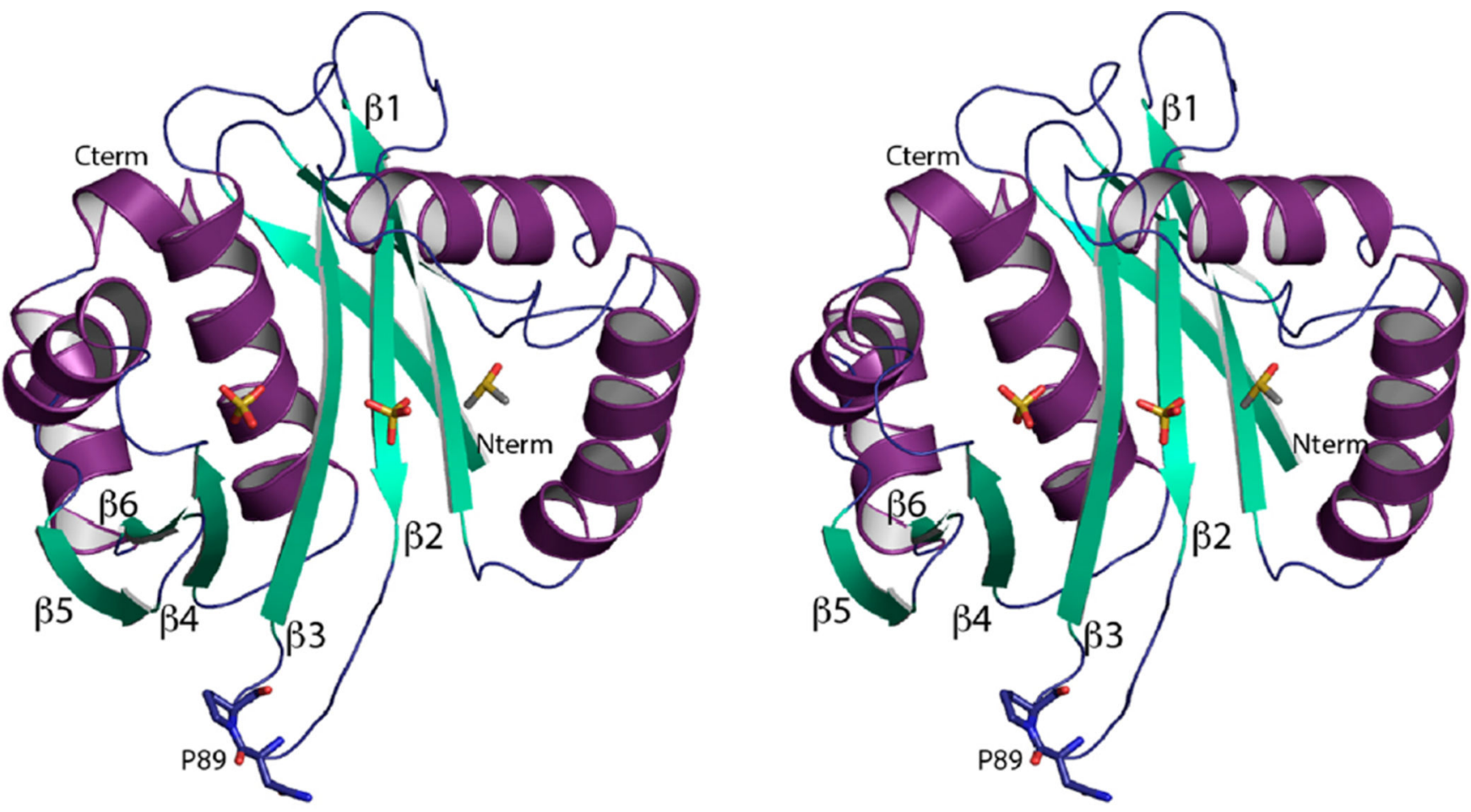

Figure 2.

Stereoribbon representation of FdhC. The $a$-helices and $\beta$-strands are colored purple and green, respectively. The sulfate anions and the dimethyl sulfoxide molecule observed binding in the interior of the protein are displayed as sticks. This figure and all others were prepared with PyMOL. ${ }^{27}$ 


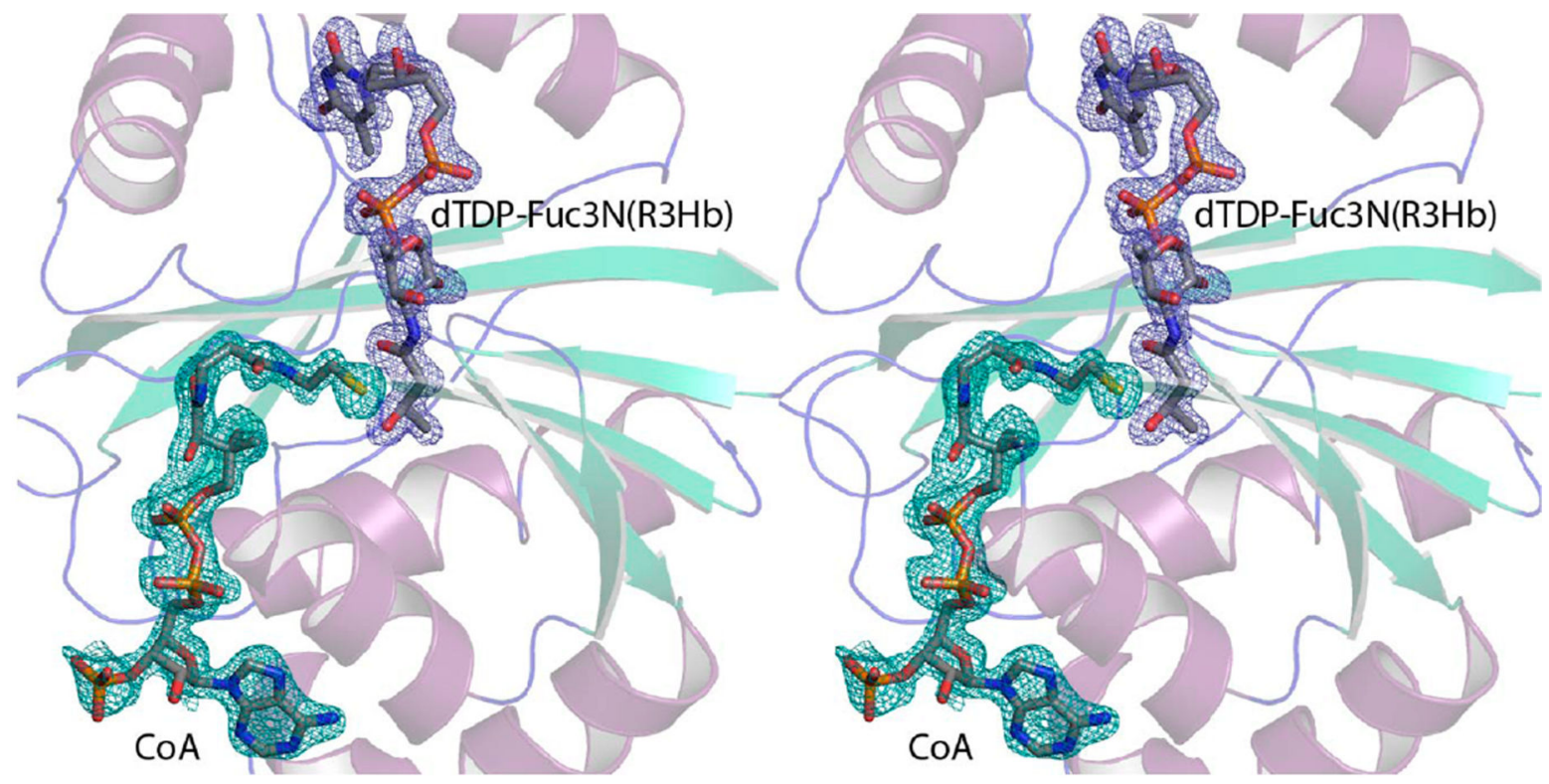

(a)

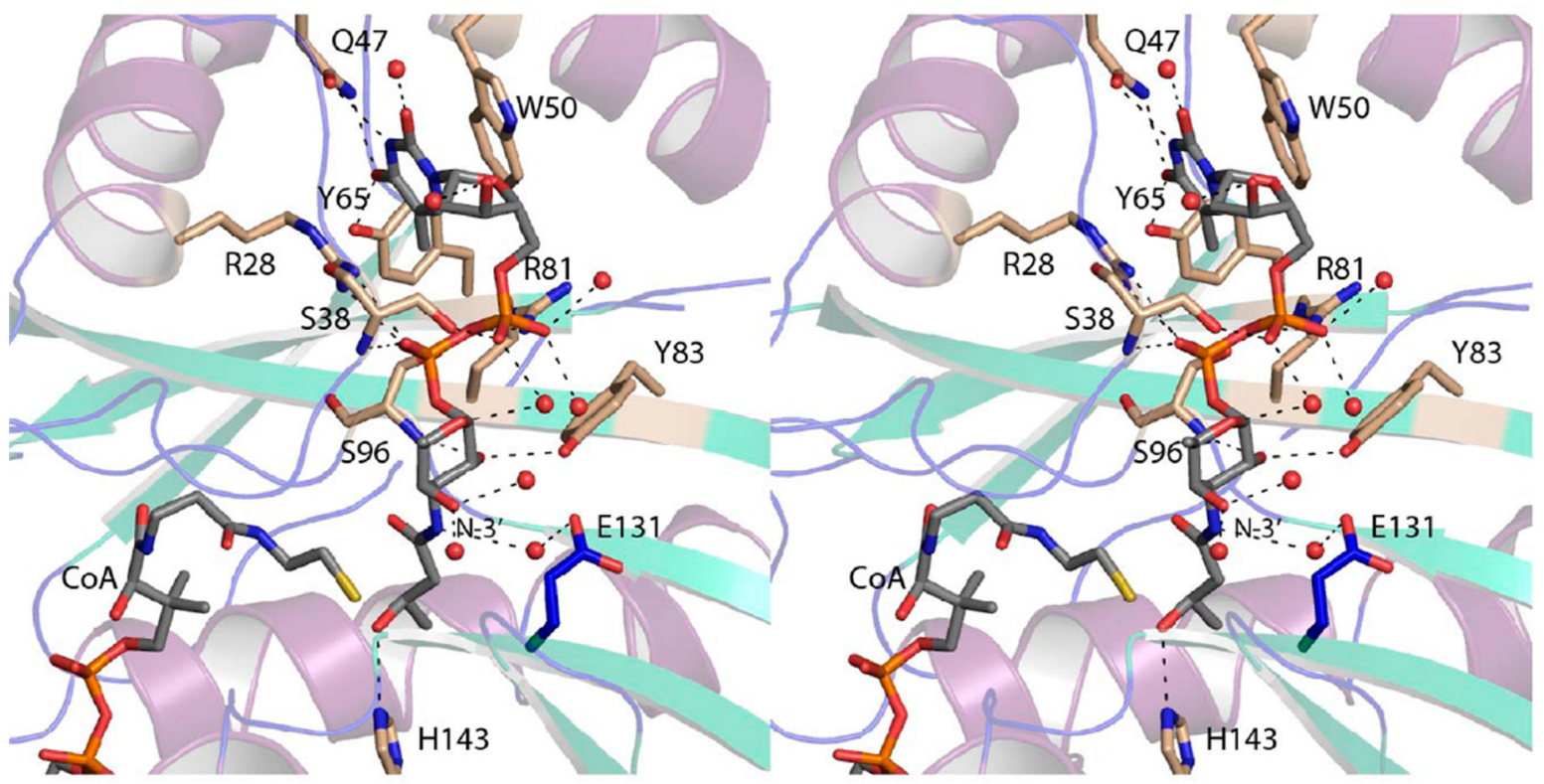

(b)

Figure 3.

Active site of FdhC. Observed electron densities corresponding to the CoA and dTDPFuc3N(R3Hb) ligands are shown in stereo in panel a. The omit map, contoured at $\sim 3 \sigma$, was calculated with coefficients of the form $F_{\mathrm{o}}-F_{\mathrm{c}}$, where $F_{0}$ was the native structure factor amplitude and $F_{\mathrm{c}}$ was the calculated structure factor amplitude. Note that the map was calculated before the ligands had been included in the X-ray coordinate file, and thus, there is no model bias. A close-up view of the region surrounding the dTDP-Fuc3N(R3Hb) ligand is presented in stereo in panel b. Possible hydrogen bonds are indicated by the dashed lines. 
The amino acid side chains that interact with the dTDP-sugar ligand are colored wheat. Glu 131 , which interacts with the amino nitrogen of the product indirectly via an ordered water molecule, is displayed in blue. 


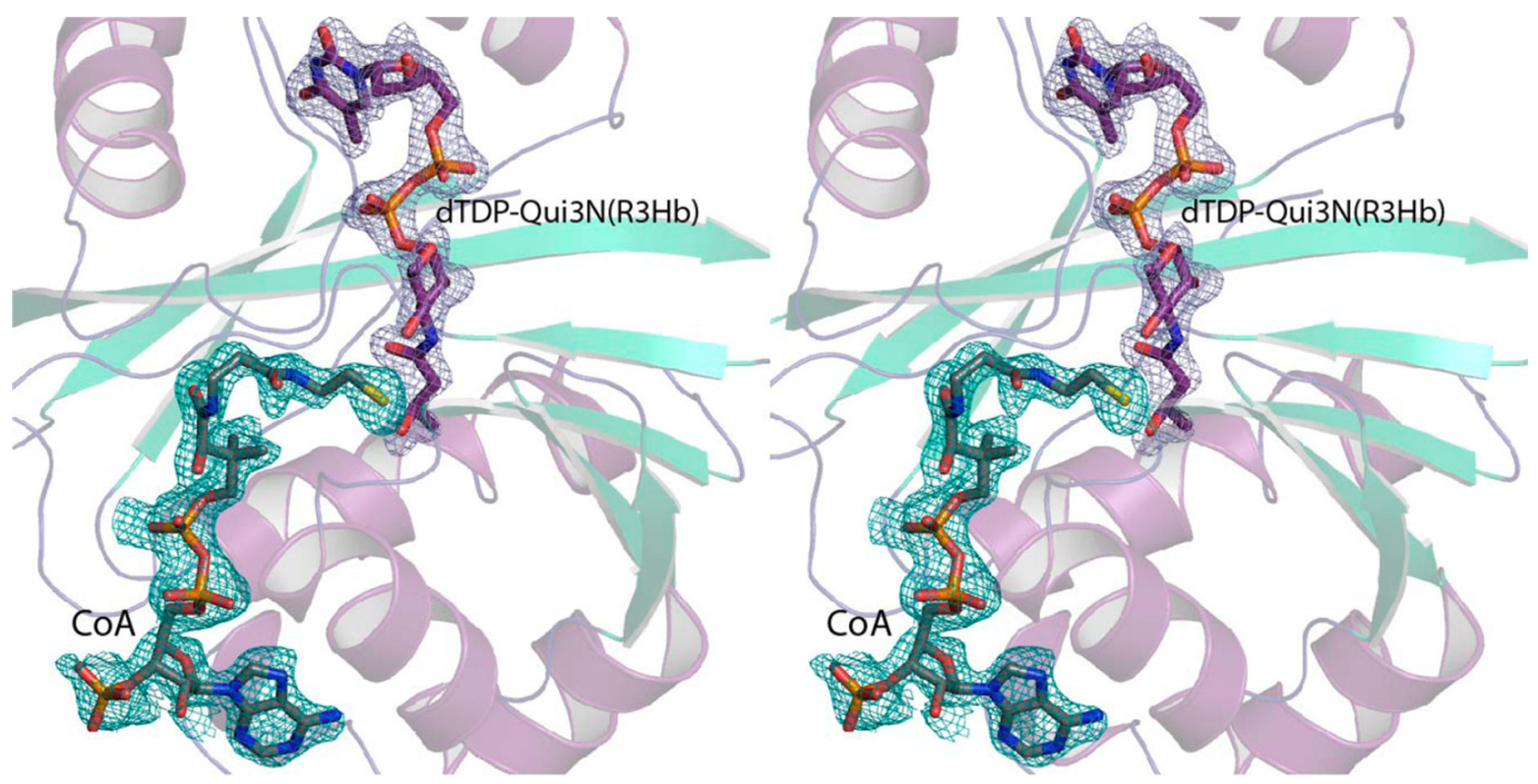

(a)

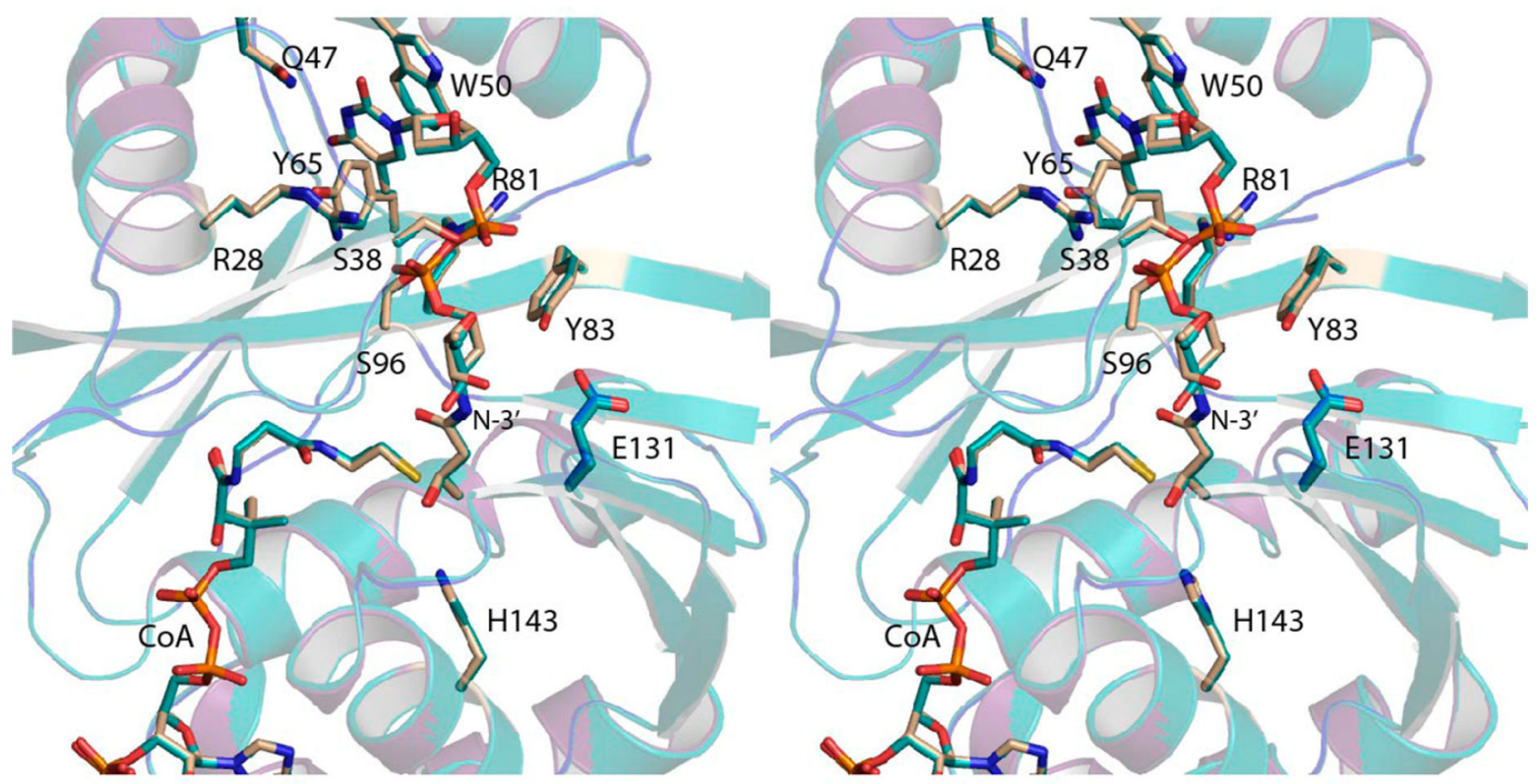

(b)

Figure 4.

Active site of FdhC with an alternative substrate. Observed electron densities corresponding to the CoA and dTDP-Qui3N(R3Hb) ligands are shown in stereo in panel a. The omit map was calculated as described in the legend of Figure 3. A superposition of the FdhC active sites with bound CoA and either dTDP-Fuc3N(R3Hb) or dTDP-Qui3N(R3Hb), colored wheat or cyan, respectively is displayed in stereo in panel $b$. 

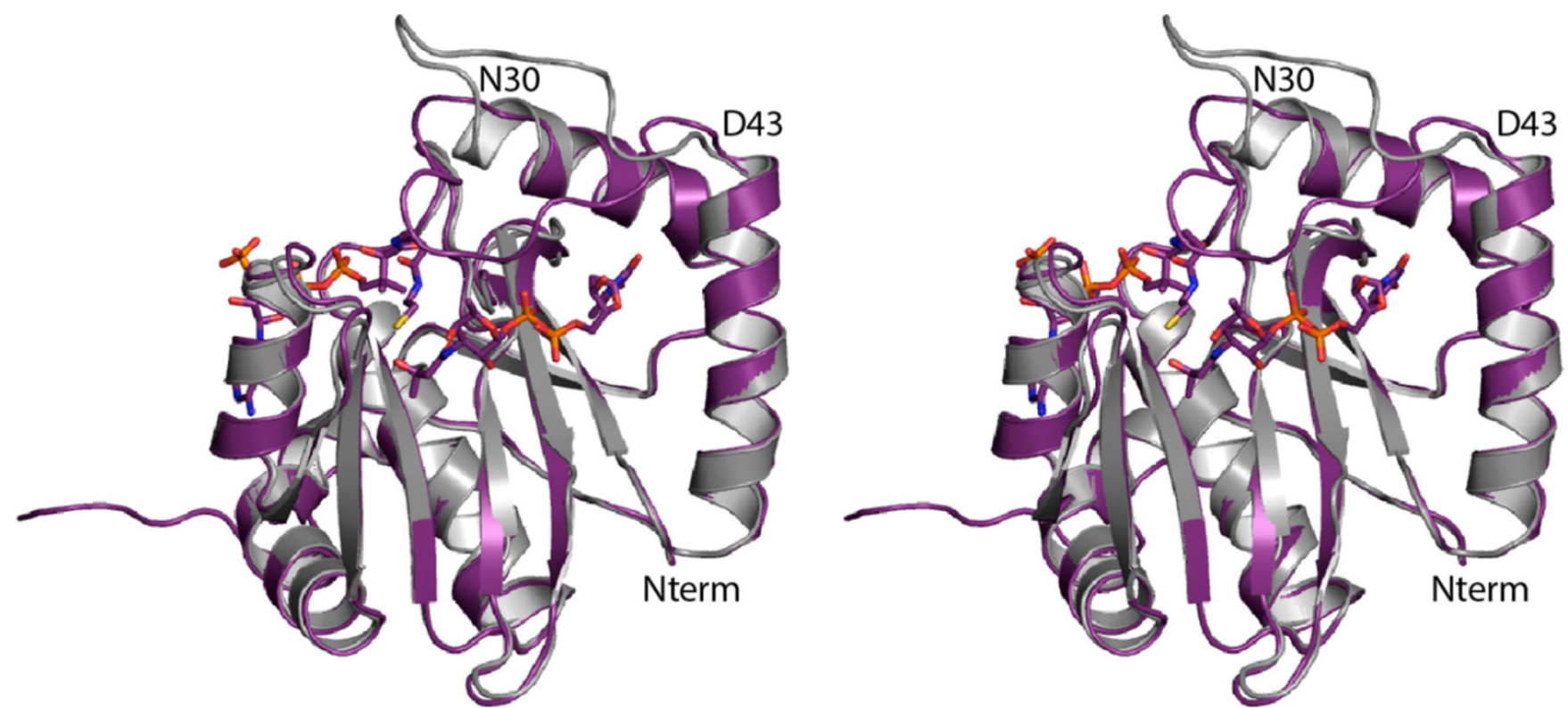

Figure 5.

Comparison of the FdhC apoenzyme and ternary complex structures. The apoenzyme is depicted in white whereas the ternary complex of FdhC/CoA/dTDP-Fuc3N(R3Hb) is drawn in violet. A dramatic difference between the two forms of the enzyme occurs in the loop conformation defined by Asn 30-Asp 43. The bound ligands are drawn as sticks. The figure is presented in stereo. 

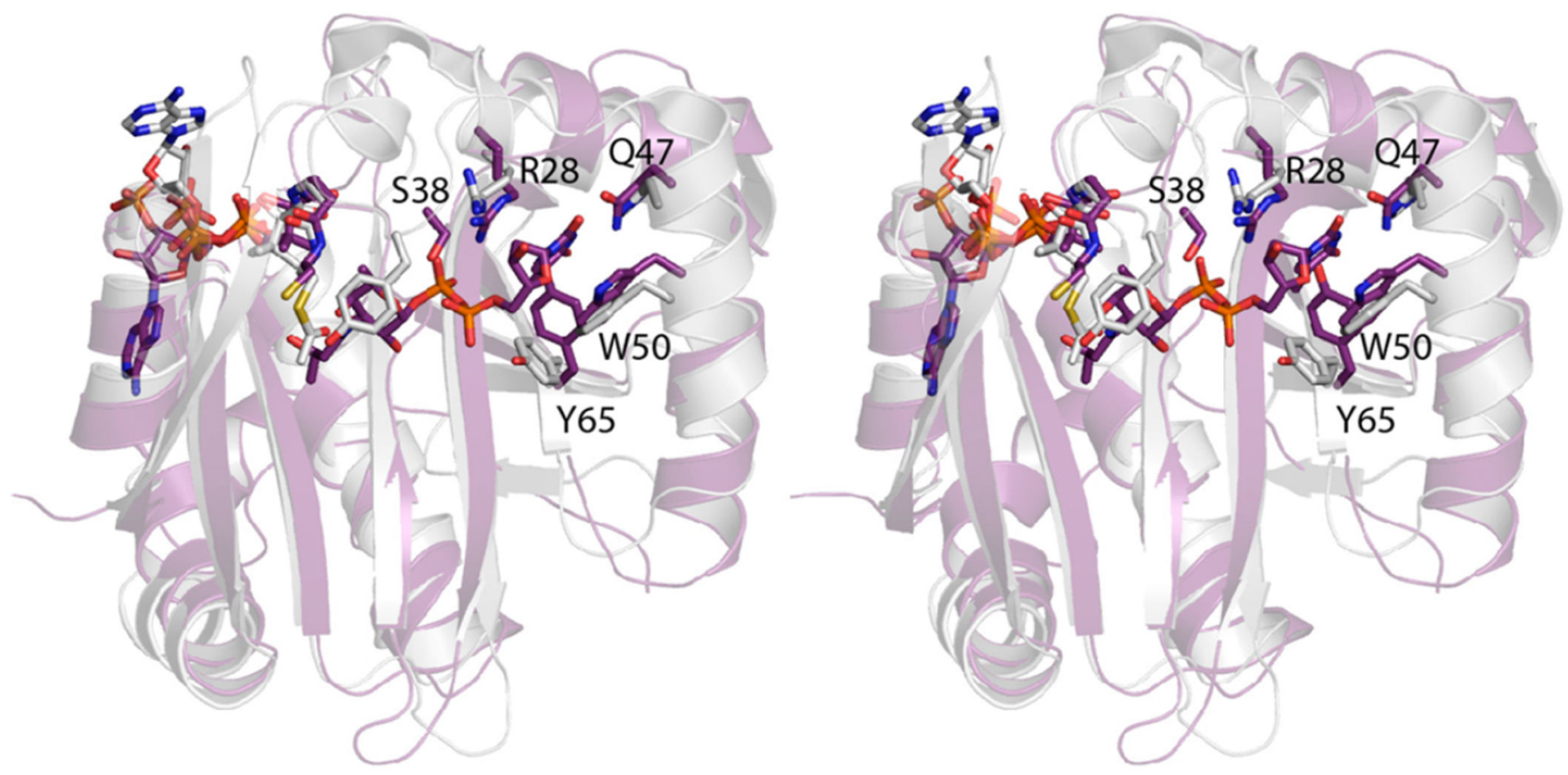

Figure 6.

Comparison of the FdhC and PseH structures. Coordinates for the PseH model were obtained from the Protein Data Bank (entry 4RI1). ${ }^{10}$ The ribbon representations for FdhC and $\mathrm{PseH}$ are displayed in violet and white, respectively. Those ligands and side chains belonging to $\mathrm{FdhC}$ are highlighted in purple where those belonging to PseH are displayed in white. The figure is presented in stereo. 
Salinger et al.

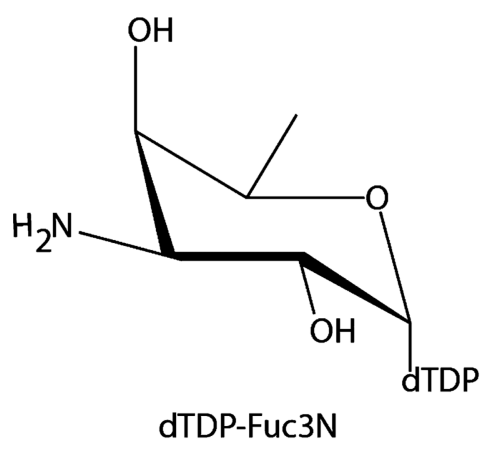

Page 20

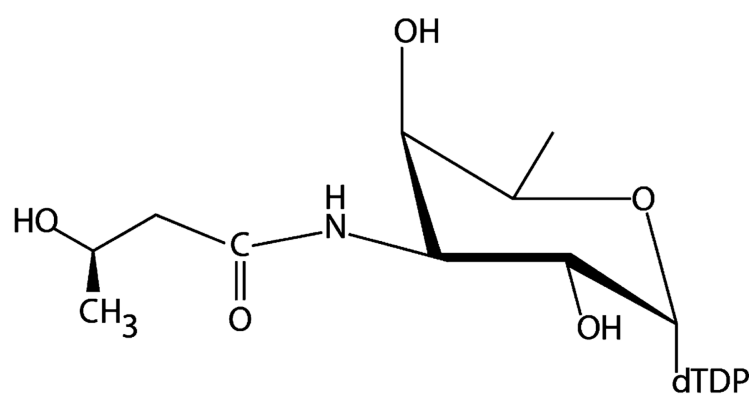

dTDP-Fuc3N(R3Hb)

Scheme 1.

\section{Reaction catalyzed by FdhC}

3-hydroxylbutanoyl-CoA 


\section{Table 1}

X-ray Data Collection Statistics

\begin{tabular}{|c|c|c|c|}
\hline & apoenzyme & $\begin{array}{l}\text { FdhC in complex with dTDP-Fuc3N(R3Hb) } \\
\text { and } \\
\text { CoA }\end{array}$ & $\begin{array}{l}\text { FdhC in complex with dTDP- } \\
\text { Qui3N }(R 3 H b) \text { and } \\
\text { CoA }\end{array}$ \\
\hline resolution limits & $50.0-1.89(1.99-1.89)^{b}$ & $50.0-1.80(1.90-1.80)$ & $50.0-1.90(2.0-1.90)$ \\
\hline $\begin{array}{l}\text { no. of independent } \\
\text { reflections }\end{array}$ & $16130(2061)$ & $20344(2775)$ & 17505 (2444) \\
\hline completeness (\%) & $98.0(93.9)$ & $98.2(91.9)$ & $99.7(98.4)$ \\
\hline redundancy & $6.9(5.1)$ & $9.5(3.8)$ & $29.5(15.4)$ \\
\hline avg $I / \operatorname{avg} \sigma(I)$ & $17.1(2.9)$ & $17.6(3.8)$ & $21.1(5.2)$ \\
\hline$R_{\mathrm{sym}}(\%)^{a}$ & $8.0(41.1)$ & $7.4(27.5)$ & $10.8(42.5)$ \\
\hline
\end{tabular}

${ }^{a} R_{\mathrm{Sym}}=(\Sigma \mid I-\bar{I} / \Sigma I) \times 100$.

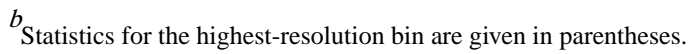




\section{Table 2}

\section{Refinement Statistics}

\begin{tabular}{|c|c|c|c|}
\hline & apoenzyme & $\begin{array}{l}\text { FdhC in complex with dTDP- } \\
\text { Fuc3N(R3Hb) } \\
\text { and CoA }\end{array}$ & $\begin{array}{l}\text { FdhC in complex with dTDP- } \\
\text { Qui3N(R3Hb) } \\
\text { and CoA }\end{array}$ \\
\hline space group & $P 6_{3} 22$ & $P 4_{1} 2_{1} 2$ & $P 4_{1} 2{ }_{1} 2$ \\
\hline unit cell dimensions $(\AA)$ & $\begin{array}{c}a=b=69.5 \\
c=137.9\end{array}$ & $a=b=76.3, c=73.5$ & $a=b=75.8, c=73.5$ \\
\hline resolution limits $(\AA)$ & $50-1.9$ & $50.0-1.8$ & $50.0-1.9$ \\
\hline$R$ factor $^{a}$ (overall) $(\%) /$ no. of reflections & $19.8 / 16107$ & $19.7 / 20303$ & $20.4 / 17457$ \\
\hline$R$ factor (working) $(\%) /$ no. of reflections & $19.5 / 15290$ & $19.4 / 19260$ & $20.0 / 16577$ \\
\hline$R$ factor (free) $(\%) /$ no. of reflections & $25.8 / 817$ & $24.7 / 1043$ & $26.9 / 880$ \\
\hline no. of protein atoms & 1486 & 1583 & 1583 \\
\hline no. of heteroatoms & 111 & 242 & 196 \\
\hline \multicolumn{4}{|l|}{ average $B$ value $\left(\AA^{2}\right)$} \\
\hline protein atoms & 24.1 & 20.6 & 23.2 \\
\hline ligands & $\mathrm{n} / \mathrm{a}$ & 19.5 & 21.5 \\
\hline solvent & 28.0 & 23.2 & 22.6 \\
\hline \multicolumn{4}{|l|}{$\begin{array}{l}\text { weighted root-mean-square deviation from } \\
\text { ideality }\end{array}$} \\
\hline bond lengths $(\AA)$ & 0.018 & 0.019 & 0.017 \\
\hline bond angles (deg) & 1.9 & 2.0 & 1.9 \\
\hline general planes (deg) & 0.009 & 0.009 & 0.008 \\
\hline \multicolumn{4}{|l|}{ Ramachandran regions $(\%)^{b}$} \\
\hline most favored & 89.1 & 90.2 & 91.3 \\
\hline additionally allowed & 10.9 & 9.2 & 8.1 \\
\hline generously allowed & - & 0.6 & 0.6 \\
\hline
\end{tabular}

${ }_{R}^{a}$ factor $=\left(\Sigma\left|F_{\mathrm{O}}-F_{\mathrm{C}} / / \Sigma\right| F_{\mathrm{O}} \mid\right) \times 100$, where $F_{\mathrm{O}}$ is the observed structure factor amplitude and $F_{\mathrm{C}}$ is the calculated structure factor amplitude.

$b$ Distribution of the Ramachandran angles according to PROCHECK. ${ }^{26}$ 
Table 3

\begin{tabular}{|c|c|c|c|c|}
\hline \multirow{2}{*}{ 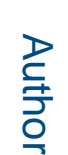 } & \multicolumn{4}{|c|}{ Kinetic Parameters for Wild-Type FdhC } \\
\hline & substrate & $K_{\mathbf{m}}(\mathbf{m M})$ & $k_{\text {cat }}\left(\mathrm{s}^{-1}\right)$ & $\begin{array}{l}k_{\text {cat }} / K_{\mathrm{m}} \\
\left(\mathbf{M}^{-1} \mathrm{~s}^{-1}\right)\end{array}$ \\
\hline 8 & dTDP-Fuc3N & $0.65 \pm 0.07$ & $52.7 \pm 2.1$ & $8.1 \times 10^{4}$ \\
\hline ह & $\begin{array}{l}\text { 3-hydroxylbutanoyl- } \\
\text { CoA }\end{array}$ & $0.69 \pm 0.11$ & $63.2 \pm 3.6$ & $9.2 \times 10^{4}$ \\
\hline 1 & dTDP-Qui3N & $5.3 \pm 0.8$ & $24.7 \pm 1.7$ & $4.7 \times 10^{3}$ \\
\hline & acetyl-CoA & $0.04 \pm 0.01$ & $0.0034 \pm 0.0002$ & $8.5 \times 10$ \\
\hline
\end{tabular}




\section{Table 4}

Kinetic Parameters for the Mutant Variants of FdhC

\begin{tabular}{lllll}
\hline & substrate & $\boldsymbol{K}_{\mathbf{m}}(\mathbf{m M})$ & $\boldsymbol{k}_{\text {cat }}\left(\mathbf{s}^{\mathbf{1}}\right)$ & $\boldsymbol{k}_{\text {cat }} / \boldsymbol{K}_{\mathbf{m}}\left(\mathbf{M}^{-\mathbf{1}} \mathbf{s}^{\mathbf{- 1}}\right)$ \\
\hline \multirow{2}{*}{ FdhC-C-term tag } & dTDP-Fuc3N & $1.5 \pm 0.2$ & $38.3 \pm 1.9$ & $2.6 \times 10^{4}$ \\
& 3-hydroxylbutanoyl-CoA & $0.20 \pm 0.04$ & $23.5 \pm 1.2$ & $1.2 \times 10^{5}$ \\
\multirow{2}{*}{ E131Q variant } & dTDP-Fuc3N & $0.24 \pm 0.19$ & $21.7 \pm 0.4$ & $9.0 \times 10^{4}$ \\
& 3-hydroxylbutanoyl-CoA & $0.64 \pm 0.08$ & $79.9 \pm 3.7$ & $1.2 \times 10^{5}$ \\
\multirow{2}{*}{ E131L variant } & dTDP-Fuc3N & $1.6 \pm 0.2$ & $23.3 \pm 1.2$ & $1.5 \times 10^{4}$ \\
& 3-hydroxylbutanoyl-CoA & $0.65 \pm 0.11$ & $22.7 \pm 1.5$ & $3.5 \times 10^{4}$ \\
\hline
\end{tabular}

